\title{
Calidad de vida de los médicos residentes del Programa de Formación de Especialistas en Pediatría
}

\author{
LUISA SCHONHAUT B. ${ }^{1}$, PAULINA ROJAS N. ${ }^{1}$, TERESA MILLÁN K. ${ }^{2}$ \\ 1. Departamento de Pediatría Campus Norte, Universidad de Chile. \\ 2. Departamento de Pediatría Campus Occidente, Universidad de Chile.
}

\begin{abstract}
Quality of life of physicians from pediatrics training programs

Background: In the quest to improve their professional opportunities and quality of life (QOL), physicians enter to Specialty Residency Training Programs. However, the process confronts them to a series of stressing factors that could, in short term, affect their health and wellbeing. Objective: To explore aspects related to professional QOL and stressing factors experienced by pediatric residents trained in public universities at Chile. Methods: A semi-structure survey was designed, validated and administered to pediatric residents from Chilean public universities, contacted in specialty meetings and visits to their training hospitals, between November 2006 and March 2007. Results: 79 pediatric residents were contacted, $65 \%$ belonging to training centers located at the Metropolitan Region and 35\% to centers from provinces. The residents were thankful for the opportunity to express their opinions. $78.5 \%$ were female and their average age was 29 years (range 25-40). 6/10 worked after hours, with an average of 18.5 hours/week and 5/ 10 worked night shifts. $50 \%$ reported physical health problems and $30 \%$ reported mental health problems, not related to the socio-demographic factors analyzed. In regard to stressing factors, $53 \%$ of residents mentioned the excessive work load related to patient care and $33 \%$ the lack of teaching and adequate supervision. In terms of career goals, $71 \%$ reported interest in continuing a subspecialty; although $50 \%$ of residents would like to work in a hospital, only $25 \%$ believe they would be able to do so. Conclusions: The current exploratory study analyzes into aspects related to QOL and mental health of pediatric residents in Chile. It would be of great interest to continue this line of research, adding the use of standardized tools and qualitative aspects, to further investigate the stressing factors and strengths of the residency training programs that could help to design and implement protective mechanisms for our residents.

(Key words: quality of life, pediatric, residency).

Rev Chil Pediatr 2009; 80 (1): 30-38
\end{abstract}

Trabajo recibido el 04 de noviembre de 2008, devuelto para corregir el 18 de noviembre de 2008, segunda versión el 21 de noviembre de 2008, aceptado para publicación el 15 de diciembre de 2008.

Correspondencia a:

Luisa Schonhaut B.

E-mail: Ischonhaut@med.uchile.cl 


\section{RESUMEN}

Introducción: En busca de mejorar las oportunidades laborales y calidad de vida (CDV), los médicos optan por los Programas de Formación de Especialistas (PFE); no obstante, este proceso los enfrenta a una serie de factores estresantes que podrían afectar, a corto plazo su salud y bienestar. Objetivo: explorar aspectos relacionados con la CDV profesional y factores estresantes de los residentes de PFE de Pediatría de las Universidades Públicas Chilenas. Metodología: Se diseñó, validó y aplicó una encuesta semiestructurada a una muestra de oportunidad de residentes de pediatría de las universidades públicas chilenas, los que fueron contactados en congresos de la especialidad y en visitas en sus centros de formación, entre noviembre/ 2006 y marzo/ 2007. Resultados: Se contactaron 79 residentes de pediatría (39,5\% del universo), $65 \%$ provenían de Centros Formadores de la Región Metropolitana 35\% de provincia. Los residentes se sintieron agradecidos de poder emitir su opinión. El 78,5\% de los encuestados era de sexo femenino, edad promedio 29 años (rango 25 a 40). Seis de cada 10 trabajaba horario extra, promedio de 18,2 horas semanales y 5 de cada 10 realizaba guardias nocturnas. La mitad declaró tener algún problema de salud física y un tercio problemas de salud mental, sin relación con los factores sociodemográficos estudiados. Respecto a los factores estresantes, $53 \%$ de los encuestados mencionó la carga asistencial excesiva y $33 \%$ la falta de docencia y de adecuada supervisión. En cuanto a la proyección laboral, 71\% refirió interés por continuar con una subespecialidad y, pese a que a uno de cada dos residentes le gustaría trabajar en hospitales, sólo uno de cada cuatro pensaba que efectivamente lo haría. Conclusiones: El presente estudio, de carácter exploratorio, nos brinda una visión sobre aspectos relacionados con la CDV y salud mental de los residentes de pediatría de nuestro país. Sin duda sería interesante continuar con esta línea de investigación, incorporando instrumentos estandarizados y aspectos cualitativos, para indagar en profundidad sobre los factores estresantes y las fortalezas de los PFE, de modo de diseñar e implementar mecanismos protectores para nuestros residentes.

(Palabras clave: calidad de vida, programa formación de especialistas, pediatría, residentes).

Rev Chil Pediatr 2009; 80 (1): 30-38

\section{Introducción}

La calidad de vida (CDV) es un concepto multidimensional, que representa la auto-percepción que tiene cada uno sobre la propia existencia. Esta percepción se construye a partir de la integración de una serie de características inherentes a la persona, como la salud física, mental y social, sumado a las expectativas y proyecto de vida; estas características se expresan a partir de la interacción con una serie de factores propios del entorno geográfico y cultural en que vive y se desenvuelve el individuo.

El adulto moderno invierte gran cantidad de horas en su desempeño profesional, probablemente ese sea el motivo por el cual la satisfacción laboral es una de las variables más importantes relacionadas con la CDV 1 . La "calidad de vida profesional" es la "experiencia de bienestar derivada del equilibrio que percibe el individuo entre las demandas o carga de trabajo desafiante, intenso y complejo, y los recursos (psicológicos, organizacionales $y$ relacionales) de que dispone para afrontar esas demandas" (García Sánchez 1993)2.

En busca de mejorar las oportunidades laborales y CDV a largo plazo, los médicos optan por los Programa de Formación de Especialistas (PFE) ${ }^{3,4}$; no obstante, este proceso los enfrenta a una serie de factores estresantes ${ }^{5}$ que podrían afectar, a corto plazo su salud y bienestar, dificultando el desarrollo de otros ámbitos de su vida ${ }^{6}$. Estudios realizados en distintas partes del mundo reportan altas tasas de problemas salud mental, como depresión ${ }^{7}$, estrés ${ }^{8}$, desgaste profesional ${ }^{9,10}$ y percepción de mala $\mathrm{CDV}^{11}$, en residentes de distintas especialidades médicas.

Para comprender y categorizar los factores estresantes a los que pueden verse sometidos el residentes durantes su estadía en los PFE se han propuesto distintas clasificaciones ${ }^{5,12,13}$, las que peden resumir como sigue:

- Factores de estrés profesional, determinado por el proceso de profesionalización, en que 
el residente asume una gran responsabilidad, la que debe compatibilizar con una importante carga académica y asistencial, habitualmente en ausencia de una adecuada supervisión docente ${ }^{14}$.

- Factores de estrés situacional o físico, derivado de los sistemas de formación de especialistas; los que históricamente han implicado una gran demanda física e intelectual, producto de largas jornadas laborales diurnas además de las guardias.

- Factores de estrés organizacional, ocasionado por una inadecuada remuneración del trabajo realizado por los residentes; un agravante de este aspecto, en nuestro sistema de formación de especialistas, se debería a que una proporción importante de residentes tiene una modalidad de autofinanciamiento de sus PFE. Otros factores a considerar son la falta de material de apoyo, instalaciones inadecuadas y las dificultades derivadas de las relaciones interpersonales en el trabajo.

- Factores de estrés personal y familiar, desencadenados por las características sociodemográficas, personalidad y experiencia de los residentes, los que están sometidos a una serie de eventos propios de la etapa del ciclo vital en la que se encuentran.

El objetivo del presente estudio es explorar aspectos relacionado con la CDV profesional y factores estresantes de los residentes del PFE de Pediatría de las Universidades Públicas Chilenas.

\section{Personas y Métodos}

El presente es un estudio corte transversal prospectivo, descriptivo y no aleatorizado.

Se obtuvo una muestra de oportunidad de residentes de pediatría, contactados en congresos de la especialidad y en visitas en sus centros de formación, durante los meses de noviembre de 2006 a marzo de 2007. El universo estimado es de 200 residentes de Pediatría de las Universidades Públicas de Chile, en el período estudiado (SOCHIPE 2008). Se excluyeron aquellos residentes que no aceptaron participar de la encuesta.
Para la obtención de la información se diseñó, validó y aplicó una encuesta semiestructurada, la que fue respondida en forma anónima, confidencial y autoadministrada, previo consentimiento informado.

La encuesta, adjuntada contaba con los siguientes ítems:

1. Información biodemográfica y laboral, recopilando aspectos relacionados con la edad, sexo, centro formador de procedencia, experiencia laboral previa, estado civil, número de hijos, personas con quienes vive, tipo de financiamiento del PFE (dividiéndose entre autofinanciados, ciclo de destinación o becas financiadas por instituciones) y situación de endeudamiento.

2. Información subjetiva, relacionada con la $\mathrm{CDV}$, la que fue categorizada a través de escala de Likert en cinco categorías. Para fines del análisis de los resultados las categorías relacionadas con el estar satisfecho y muy satisfecho además de siempre y casi siempre, se agruparon como de satisfecho y siempre, respectivamente, por otro lado, las categorías de muy insatisfecho e insatisfecho y nunca o casi nunca se agruparon como insatisfecho y nunca, respectivamente.

3. Preguntas abiertas referidas a los aspectos que más les agradaban y disgustaban del PFE.

El análisis estadístico se realizó a través de estadísticas descriptivas (promedios, modas, medianas). Se analizó la asociación entre problemas de salud mental y factores biodemográficos, a través de prueba de $\chi^{2}$. Dado que es una muestra de oportunidad, no permite inferencia estadística, pero sí se pueden observar tendencias de asociación entre las variables estudiadas.

\section{Resultados}

\section{Características de los sujetos estudiados}

Se obtuvo 79 encuestas, correspondientes al $39,5 \%$ del universo de residentes. Cincuenta y uno $(65 \%)$ provenían de Centros Formadores de la Región Metropolitana de Chile y 28 (35\%) 
de Centro Formadores de provincia (quinta región y sur de Chile), lo que corresponde a una proporción similar a la distribución del universo de residentes.

No hubo rechazos por participar de la encuesta, por el contrario, los residentes que se contactaron se sintieron muy agradecidos de poder emitir su opinión.

"Me parece excelente que alguien se preocupe de hacer algo por la salud mental y emocional de los residentes".

"Ojala que con la encuesta logren cambios positivos!!".

"Gracias y jauxilio!".

\section{Características biodemográficas}

Las características biodemográficas se muestran en la en la tabla 1. Sesenta y dos residentes eran de sexo femenino (78,5\%). La edad promedio fue de 29 años, rango 25 a 40 y moda de 26 años.

Los años promedio de egreso de la carrera de Medicina fueron 3,5, con un máximo de 10 años. La mitad de los encuestados tenía experiencia laboral previa al inicio del PFE de Pediatría.

En relación al estado civil, $38 \%$ de los residentes tenía pareja estable. Durante la estadía en el PFE, 3 médicos contrajeron matrimonio y uno se separó. Veintidos residentes $(28 \%)$ tenían hijos, la mayoría uno, siendo el máximo de 3 hijos.

\section{Perfil laboral}

Setenta por ciento de los residentes sentía que estaba trabajando en exceso, y $90 \%$ falta de sueño: "iSi tengo algún tiempo libre lo ocupo en dormir!'. Cuarenta y siete (59,5\%) de los residentes entrevistados trabajaba además del horario del programa, con un promedio de 18,2 horas semanales, rango 4 a 40, moda 10 horas, 42 de ellos hacía un turno de guardia nocturna además de la guardia exigida por el PFE. No se encontró asociación entre el trabajar horario extraprogramático con la condición de provisión del grupo familiar o endeudamiento.

\section{Estilos de vida}

Manifestaron cambios negativos en los hábitos alimenticios $78 \%$, especialmente debido a desorden en los horarios y calidad de la alimentación. Cincuenta $(63,3 \%)$ había sufrido cambios significativos en el peso corporal, 31 habían subido y 19 bajado de peso. En la figura 1 se muestran los estilos de vida y hábitos de los residentes.

La gran mayoría, 96\%, manifestó que producto de su participación en el PFE la cantidad de tiempo libre y tiempo dedicado a actividades personales y familiares se había deteriorado, $72 \%$ percibía que además la cantidad, la calidad del tiempo dedicado a esas actividades, se había comprometido negativamente. "Creo que vale la pena vivir sola, alejada de los que amo, para lograr ser pediatra, a pesar de exigir un gran esfuerzo y lucha diaria".

Tabla 1. Características biodemográficas de los residentes encuestados

\begin{tabular}{|c|c|c|c|c|c|c|}
\hline Característica & Distribución & (\%) & Distribución & (\%) & Distribución & (\%) \\
\hline Sexo & Femenino & 78,5 & Masculino & 21,5 & & \\
\hline Procedencia & Región Metropolitana & 66,0 & Provincia & 34,0 & & \\
\hline Año residencia & Primero & 40,0 & Segundo & 32,0 & Tercero & 28,0 \\
\hline Experiencia laboral & $\mathrm{Si}$ & 50,0 & No & 50,0 & & \\
\hline Situación conyugal & Con pareja & 38,0 & Soltero & 62,0 & & \\
\hline Hijos & $\mathrm{Si}$ & 28,0 & No & 72,0 & & \\
\hline Vive con sus padres & $\mathrm{Si}$ & 39,0 & No & 44,0 & & \\
\hline Proveedor & $\mathrm{Si}$ & 48,0 & No & 50,0 & & \\
\hline Financiamiento & Autofinanciado & 72,0 & Ciclo destinación & 20,0 & Becado & 8,0 \\
\hline Endeudamiento & $\mathrm{Si}$ & 53,0 & No & 46,0 & & \\
\hline
\end{tabular}




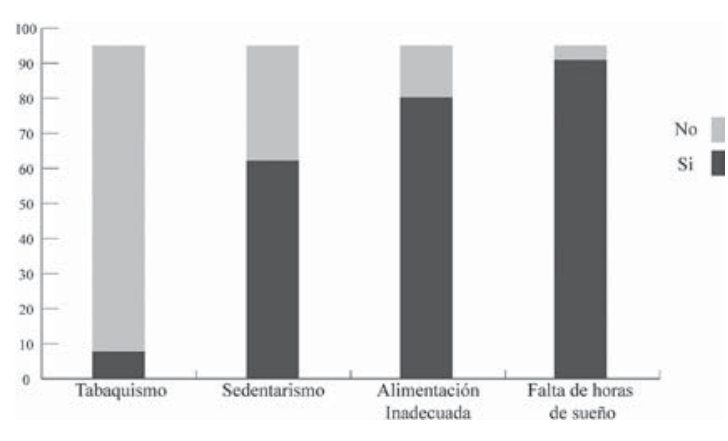

Figura 1. Estilos de vida y hábitos.

\section{Estado de ánimo}

En la figura 2 se ilustran los cambios en los estados de ánimo manifestados por los residentes durante su estadía en el PFE, información obtenida comparando el "antes" de comenzar el PFE con el "durante" el mismo. Al respecto destaca que el porcentaje de residentes que refería sentirse "nunca deprimido" antes de comenzar el PFE era de 75\%, cifra que disminuyó al 38\% durante el programa de residencia. Por otro lado, el porcentaje de residentes se sentían estresados aumentó del 7,6\% a 46,8\% desde el inicio del PFE.

\section{Salud mental y factores relacionados}

Uno de cada dos residentes mencionó la presencia de algún problema de salud física, la mitad de ellos podría deberse a patologías de origen psicosomático: 13 problemas neuromusculares como lumbago y otras mialgias, 8 cefaleas y 4 trastornos gastrointestinales.

Uno de cada tres declaró problemas de salud mental no especificado, y, de ellos, la mitad se encontraba en tratamiento. No se encontró asociación con factores personales estudiados ni tampoco con factores físicos $\mathrm{u}$ organizacionales, como el cansancio, trabajo en exceso, satisfacción con el PFE ni con el ambiente laboral.

Hubo asociación entre problemas declarados de salud mental y estilos de vida menos saludables, como la adquisición de hábitos negativos en la alimentación $(\mathrm{p}=0,05)$ y cambios significativos en el peso $(p=0,026)$.

En cuanto a la proyección futura, los residentes con problemas declarados de salud mental refirieron mayor intención de interrumpir temporalmente el PFE $(p=0,018)$, pero no hubo diferencias en cuanto a la intención por continuar con una subespecialidad.

\section{Satisfacción con el PFE de Pediatría}

En la figura 3 se ilustra la satisfacción de los residentes con algunos aspectos del PFE de Pediatría, destacando la relación con los com-

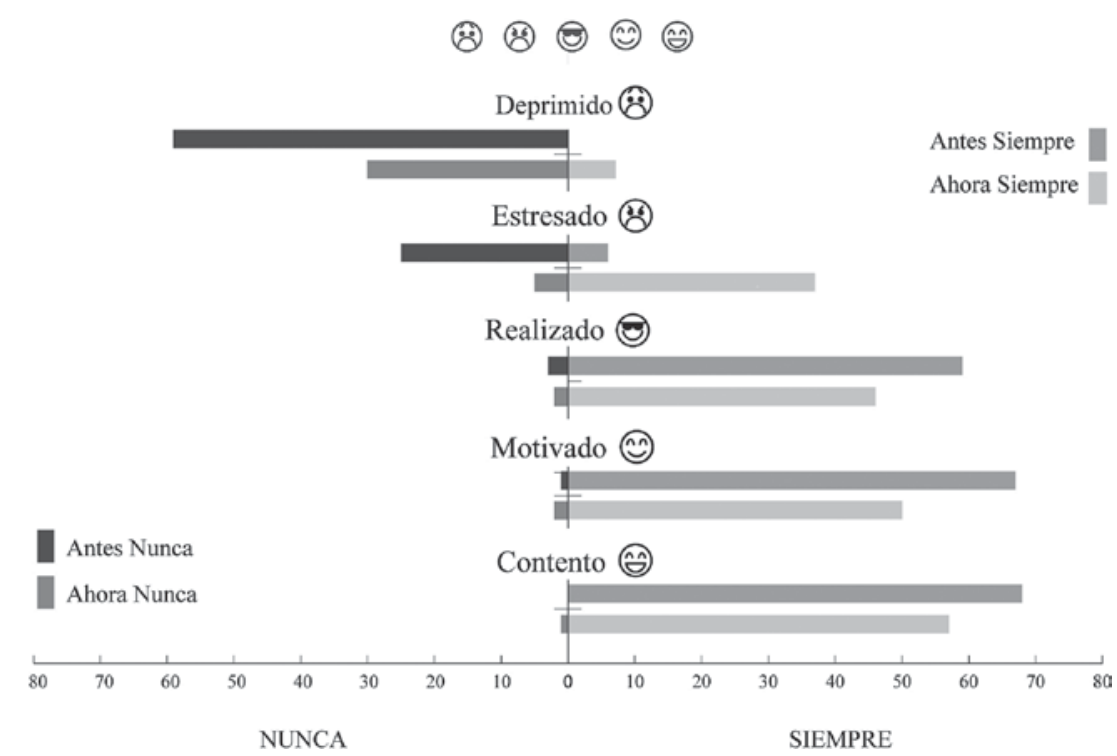

Figura 2. Cambio de ánimo de becados. 


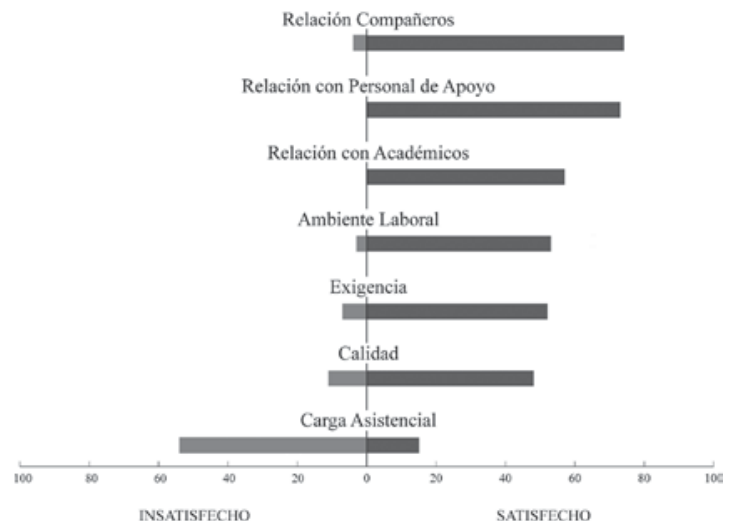

Figura 3. Satisfacción con el PEEP. pañeros (94\%), relación con personal de apoyo $(92 \%)$, relación con académicos $(72 \%)$, ambiente laboral (67\%), exigencia (66\%), calidad $(61 \%)$ y alto grado de insatisfacción con la carga asistencial (68\%).

En la tabla 2 se clasifican las respuestas obtenidas respecto a la pregunta abierta sobre los aspectos que más les gustaban y disgustaban del PFE.

\section{Expectativas laborales}

Respecto a las expectativas laborales futuras, $54 \%$ respondió en forma optimista y $26 \%$ veía un futuro incierto. Cincuenta y seis $(71 \%)$

Tabla 2. Respuestas obtenidas respecto a la pregunta abierta sobre los aspectos que más les gustaban y disgustaban del PFE

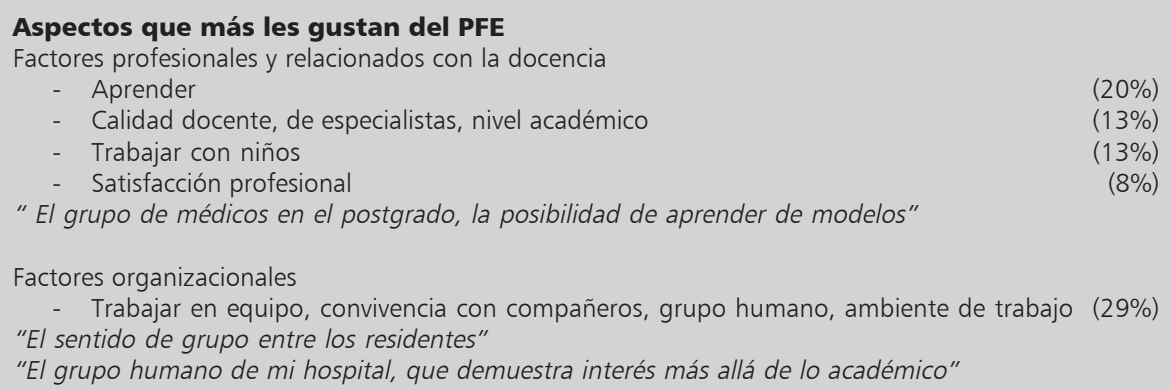

"El grupo de médicos en el postgrado, la posibilidad de aprender de modelos"

Factores organizacionales

Trabajar en equipo, convivencia con compañeros, grupo humano, ambiente de trabajo (29\%)

"El sentido de grupo entre los residentes"

"El grupo humano de mi hospital, que demuestra interés más allá de lo académico"

\section{Aspectos que más les disgustan del PFE}

Factores profesionales y relacionados con la docencia

Carga asistencial excesiva y sentirse utilizado

Falta de docencia y de adecuada supervisión

Mal trato a los residentes

Falta de reconocimiento

"La carga asistencial sobrepasa las instancias académicas."

"El trabajo del residente no es reconocido, es tomado como una obligación asistencial."

"Nos ven como mano de obra y no como colegas"

"En ocasiones me han tratado como auxiliar administrativo y no como médico"

"Me disgusta que nos reten en público (lo merezcamos o no)"

Factores situacionales o físicos

- Falta de tiempo para estudiar

Falta de tiempo libre

"El mayor deterioro en la calidad de vida se debe al hecho de vernos obligados a trabajar en horarios no hábiles, haciendo guardias, para poder costear el programa."

Factores organizacionales

Inadecuada infraestructura

Altos aranceles y trabajo asistencial no remunerado

Factores de conciliación personal y familiar

"Es difícil estar en esta etapa de formación, cuando uno además está en etapa de plena crianza y de formación familiar."

"Falta preocupación por otros aspectos del residente como la salud física, psicológica, situación financiera, etc." 


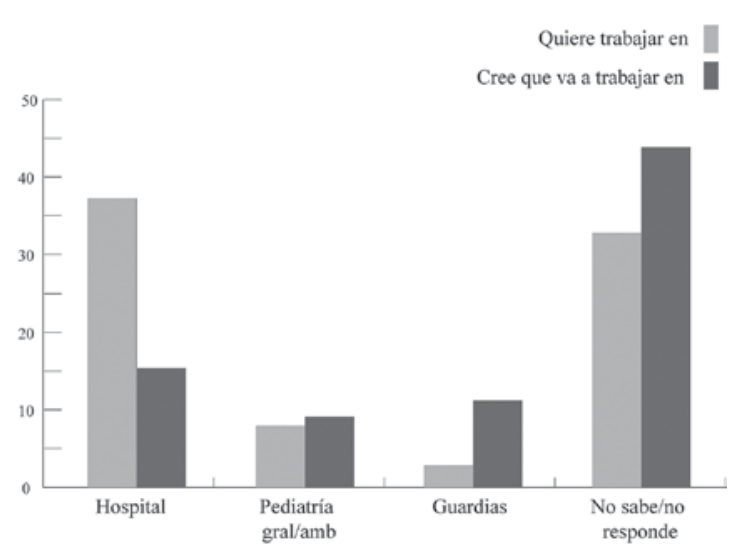

Figura 4. Expectativas laborales de los residentes de pediatría.

manifestaron interés por continuar con una subespecialidad. En la figura 4 se muestra la brecha entre las expectativas laborales de los residentes, es decir, entre el lugar en que les gustaría trabajar versus el lugar donde creen que trabajarían, observándose que a 45,5\% le gustaría trabajar en hospitales, sólo la mitad de ellos $(20 \%)$ creía que efectivamente lo haría.

\section{Discusión}

El presente estudio fue diseñado con carácter exploratorio, con el propósito de conocer la CDV de los residentes de pediatría del país. Se pensaba que se encontrarían diferencias entre las vivencias de los residentes de provincia en comparación a los santiaguinos, que tendría relevancia el tipo de financiamiento de PFE, la situación de endeudamiento o la necesidad de trabajar en horarios extra... pero no fue así, independiente de los factores analizados, un porcentaje importante de los residentes "no lo estaba pasando bien" en esta etapa de su vida; más aún, los entrevistados/as se sintieron agradecidos/as de tener la oportunidad de expresar en forma anónima sus sentimientos y percepciones. "Gracias y iauxilio!".

Al indagar por distintos aspectos relacionados con la CDV, destaca que la mayoría refirió estar cansado/a y trabajar en exceso, independiente de si trabajaban o no horas extra programáticas; un alto porcentaje manifestó estar menos contento/a, motivado/a o realizado/a y más estresado/a y deprimido/a desde que comenzó el PFE; pero, lo que resulta aún más alarmante, es que uno de cada tres residentes declaró tener problemas de salud mental y uno de cada dos problemas de salud física. En el análisis estadístico no se encontró asociación con los factores biodemográficos estudiados.

La carga excesiva de trabajo, especialmente debido a las guardias, provocaría una falta "crónica" de sueño y de tiempo libre, lo que explicaría en gran medida el deterioro del ánimo, de la salud y CDV de los médicos/as residentes; ${ }^{11,15}$ pero además afectan las relaciones del equipo de salud ${ }^{16}$ y se asocian a un aumento en el riesgo de errores médicos ${ }^{9}$.

Por este motivo, a lo largo de las últimas décadas, los PFE han sido sometidos a críticas en diversos países y se ha normado el horario de trabajo de los residentes ${ }^{17,18}$. Quizás sería el momento de revisar en profundidad esta realidad en nuestro país, en que la jornada laboral exigida por el PFE en Pediatría es de 40 a 70 horas semanales, a las que habría que sumar las horas extraordinarias, realizada por más de la mitad de los residentes, en rangos de hasta 40 horas semanales.

Si bien la frecuencia de insatisfacción con la CDV y los problemas de salud mental identificados en nuestro estudio están dentro de los rangos descritos en la literatura ${ }^{8,11}$, probablemente se han subestimado debido a que formulamos preguntas generales; para conocer la real prevalencia del problema en estudio, habría que aplicar instrumentos estandarizados e indagar en profundidad sobre las percepciones de los residentes y las fuentes de estrés, mediante metodologías cualitativas.

$\mathrm{Al}$ analizar los factores estresantes emanados de la encuesta, podemos destacar que, en general, hubo un alto grado de satisfacción con los PFE, en cuanto a la calidad y exigencia, pero no así en relación a la carga asistencial, asociada a una excesiva responsabilidad, falta de adecuada supervisión y compromiso docente, aspecto descrito en otras publicaciones ${ }^{19,20}$ "Nos ven como mano de obra y no como colegas". Sumado a esto, habría que considerar la presión por trabajar para financiar el PFE, "el mayor deterioro en la calidad de 
vida se debe al hecho de vernos obligados a trabajar en horarios no hábiles, haciendo guardias, para poder costear el programa".

En cuanto a las expectativas laborales, siete de cada diez encuestados manifestó interés por continuar con una sub-especialización; $y$, a pesar que uno de cada dos residentes quisiera continuar trabajando en hospitales, sólo la mitad de ellos lo veía como una real opción laboral; esta diferencia y quizás también la incertidumbre frente al futuro laboral, podría ser reflejo de la orientación de los actuales PFE en Pediatría, focalizados en la alta complejidad hospitalaria, no obstante que el campo de desempeño profesional está cada vez más centrado en la pediatría ambulatoria ${ }^{21}$.

Los PFE en pediatría enfrentan nuevos desafíos y deberían replantearse de acuerdo a la realidad epidemiológica, demográfica, social y laboral, considerando además la tendencia en Educación en Ciencias de la Salud ${ }^{22}$, pero por sobre todo, deberían centrarse en los residentes, considerar su proyecto de vida actual y futuro, CDV, salud e inquietudes.

\section{Conclusiones}

El presente estudio, pionero en nuestro país, nos brinda una visión sobre los problemas en relación a la CDV y salud mental de los residentes de Pediatría de nuestro país.

A partir de los resultados obtenidos, podemos destacar que la mitad de los residentes de pediatría declaró problemas de salud física y un tercio problemas de salud mental, a pesar de lo alarmante de dichas cifras, llama la atención que un porcentaje significativo de los encuestados "no lo está pasando bien" por sentirse más deprimido, estresado y menos contento desde que comenzó el programa de residencia. El 96\% manifestó que durante su participación en el PFE se había visto afectada la cantidad y calidad de tiempo libre familiar y personal.

$\mathrm{Al}$ analizar los factores estresantes, destacan los de índole profesional y académico, ya que $53 \%$ de los encuestados mencionó espontáneamente la carga asistencial excesiva y 33\% la falta de docencia y de adecuada supervisión. El 90\% de los residentes refirió falta de sueño y
$70 \%$ sentía que trabajaba en exceso, a pesar que el $59,5 \%$ trabajaba horas extraprogramáticas, la mayoría haciendo guardias nocturnas. Entre los aspectos positivos de los PFE de pediatría destaca el ambiente laboral, la relación con los compañeros, personal de apoyo y con los académicos.

Sin duda sería interesante continuar con esta línea de investigación, incorporando instrumentos estandarizados y aspectos cualitativos, para indagar en profundidad sobre los factores estresantes y analizar las fortalezas personales y de algunos PFE, de modo de implementar mecanismos protectores, ya sea replanificando la carga laboral, el modo de financiero y reforzando las redes de apoyo de los residentes.

\section{Referencias}

1.- Fernández Sanmartín MI, Villagrasa Ferrer JR, Fe Gamo M, et al: Estudio de la satisfacción laboral y sus determinantes en los trabajadores sanitarios de un área de Madrid. Rev Esp Salud Pública 1995; 69: 487-97.

2.- García Sánchez S: La qualitat de vida professional com a avantatge competitiu. Revista de Qualitat 1993; 11: 4-9.

3.- Horwitz N: El cambio de la práctica Médica. Desafíos psicosociales para la profesión. Rev Méd Chile 2004; 132: 768-72.

4.- Newton D, Garayson M, Thompson L: The variables influence of lifestyle and income on medical students' career specialty choices: data from two U.S. Medical Schools, 1998-2004. Academic Medicine 2005; 80: 809-14.

5.- Nogueira-martins LA, Jorge MR: Natureza e magnitude do estresse na Residência Médica. Rev Assoc Med Bras 1998; 44: 28-34.

6.- Ratanawongsa N, Wright SM, Carrese JA: Well-being in residency: a time for temporary imbalance?. Med Educ 2007; 41: 273-80.

7.- Martínez-Lanz P, Medina-Mora E, Rivera E: Adicciones, depresión y estrés en médicos residentes. Rev Fac Med (Mex) 2005; 48: 191-7.

8.- Cohen J, Patten S: Well-being in residency training: a survey examining resident physician satisfaction both within and outside of residency training and mental health in Alberta. BMC Med Educ 2005; 5: 21.

9.- Fahrenkopf A, Sectish T, Barger L, et al: Rates of medication errors among depressed and burnt out residents: prospective cohort study. BMJ 2008 336: 488-91.

10.- Ordenes DN: Prevalencia de Burnout en trabajadores del hospital Roberto del Río. Rev Chil Pediatr 2004; 75: 449-54.

11.- Fernández O, Moreno S, Hidalgo C, García Del Río 
B, Martín A: Percepción de la calidad de vida profesional de los médicos residentes de dos hospitales de distinto nivel asistencial. Medicina de Familia 2007;7 (2) Disponible en www. [accedido 10 de julio 2008] http://archivosdemedicina.com/ojs/index.php/archmed/ article/viewFile/28/75

12.- Levey RE: Sources of Stress for Residents and Recommendations for Programs to Assist Them. Academic Medicine 2001 76: 142-50.

13.- Richardson-López Collada V: Y a nuestros residentes ¿quién los cuida?. Bol Med Hosp Infant Mex 2006; 63: 155-7.

14.- Schonhaut L: ¿Es necesario replantear el programa de postítulo de pediatría con mayor énfasis a la pediatría ambulatoria?: Perfil de competencias del residente de pediatría 2006. Universidad de Chile. Tesis para optar al título de Magíster Educación en ciencias de la Salud. Escuela de Postgrado. Facultad de Medicina. Universidad de Chile 2007.

15.- Méndez-Gonzáles M, Ryan P, García B: Efecto subjetivo de las guardias sobre la salud, CDV y calidad asistencial en residentes de España. Archivos de medicina 2005; 1: 1. Disponible en www [accedido 11 de julio 2008] http://dialnet.unirioja.es/servlet/articulo? codigo $=1057778$.

16.- Ratanawongsa N, Wright SM, Carrese JA: Well-being in residency: Effects on relationships with patients, interactions with colleagues, performance, and motivation. Patient Educ Couns 2008 72: 194-200.

17.- Report of the ACGME Work Group on Resident Duty Hours and the Learning Environment. Chicago, Ill: Accreditation Council for Graduate Medical Education; 2002. http://www.acgme.org

18.- Philibert I, Friedmann $P$, Williams $W$, et al: New Requirements for Resident Duty Hours. JAMA.2002; 288: 1112-4.

19.- Frayer-Edwards K, Wilkins D, Baernstein A, Braddock $C$ : Bringing ethics education to the clinical years: ward ethics sessions at the University of Washington. Academic Medicine 2006; 81: 626-31.

20.- Hicks L, Lin Y, Robertson D, Robinson D, Woodrow $S$ : Understanding the clinical dilemmas that shape medical students' ethical development: questionnaire survey and focus group study. BMJ 2001; 322: 709710. [Consultado 10 octubre 2006] Disponible en World Wide Web: http://bmj.bmjjournals.com/cgi/ content/full/322/7288/709.

21.- Schonhaut L, Millán T, Hanne C: Formación de especialistas en pediatría y su adecuación a la realidad epidemiológica y laboral. Rev Chil Pediatr 2007; 78: 599-606.

22.- Schonhaut L: Los desafíos de la enseñanza de pediatría ambulatoria en el contexto del chile actual. Rev Chil Pediatr 2006; 77: 405-11. 